\title{
Restoration actions in marine ecosystems: a global analysis
}

Simonetta Fraschetti ${ }^{1}$, Giuseppe Guarnieri ${ }^{1}$, Loredana Papa ${ }^{1}$, Chris $\mathrm{McOwen}^{2}$, Nadia Papadopoulou $^{3}$, Meri Bilan ${ }^{4}$, Christoffer Boström ${ }^{5}$, Pol Capdevila ${ }^{6}$, Laura Carugati ${ }^{7}$, Emma Cebrian $^{8}$, Thanos Dailianis ${ }^{3}$, Francesco De Leo ${ }^{1}$, Dario Fiorentino ${ }^{9}$, Karine Gagnon ${ }^{5}$, Cristina Gambi $^{7}$, Joaquim Garrabou ${ }^{10}$, Vasilis Gerovasileiou ${ }^{3}$, Bernat Hereu ${ }^{6}$, Silvija

Kipson ${ }^{11}$, Jonne Kotta ${ }^{12}$, Jean-Baptiste Ledoux ${ }^{10}$, Cristina Linares ${ }^{6}$, Juliette Martin ${ }^{2}$, Alba Medrano $^{6}$, Ignasi Montero-Serra ${ }^{6}$, Telmo Morato ${ }^{4}$, Antonio Pusceddu ${ }^{13}$, Katerina Sevastou ${ }^{3}$, Chris Smith ${ }^{3}$, Jana Verdura ${ }^{8}$, Roberto Danovaro ${ }^{14}$

${ }^{1}$ Department of Biological and Environmental Science and Technology, University of Salento CoNISMa, Lecce, Italy

${ }^{2}$ UN Environment World Conservation Monitoring Centre, Cambridge, United Kingdom

${ }^{3}$ Hellenic Centre for Marine Research, Heraklion, Greece

${ }^{4}$ IMAR-UAz, Instituto do Mar Centro da Universidade dos Açores, Azores, Portugal

${ }^{5}$ Environmental and Marine Biology Faculty of Science and Engineering, Åbo Akademi University, Turku/Åbo, Finland

${ }^{6}$ University of Barcelona, Department of Evolutionary Biology, Ecology and Environmental Sciences, Barcelona, Spain

${ }^{7}$ Dipartimento di Scienze della Vita e dell'Ambiente, Università Politecnica delle Marche, Ancona, Italy

${ }^{8}$ Institute of Aquatic Ecology Department of Environmental Sciences, University of Girona, Girona, Spain

${ }^{9}$ Helmholtz Institute for Functional Marine Biodiversity, University Oldenburg, AlfredWegener-Institut, Helmholtz Center for Polar and Marine Research, Oldenburg, Germany

${ }^{10}$ CSIC, Institut Ciències del Mar, Barcelona, Spain

${ }_{11}^{11}$ Department of Biology, Faculty of Science, University of Zagreb, Zagreb, Croatia

${ }^{12}$ Estonian Marine Institute, University of Tartu, Tartu, Estonia

${ }^{13}$ Dipartimento di Scienze della Vita e dell'Ambiente, Università di Cagliari, Cagliari, Italy

${ }^{14}$ Stazione Zoologica di Napoli, Università Politecnica delle Marche, Ancona, Italy

Corresponding Author

Simonetta Fraschetti

simona.fraschetti@unisalento.it

A review of 573 studies on active restoration actions in the marine environment, published in the last 25 years, was carried out at global scale. We assessed how, where, at which spatial and temporal scales and under which socio-ecological settings restoration studies have been carried out, from very shallow to deep sea habitats. Results show that restoration efforts across habitats are increasing, especially in seagrasses and coral reefs, but never approached at ecosystem level. Targets, methods, response variables and standards are still very heterogeneous. Of the factors considered in the review, habitat, human impact intensity, realm and methods of restoration were found to be good determinant of restoration success. Short project duration (one-two years), small restoration areas $(<1 \mathrm{ha})$, lack of controls and knowledge of baselines are still a limit for deriving generalities. Finally, restorations rarely consider future challenges linked to global change this impairing long-term success stories. Restoration science needs more robust 
approaches leading to the development of best practices (e.g. protocols, monitoring of the effects, reasons for failure) to be applied at spatial and temporal scales so as to answer to present and future disturbance regimes.

Marine restoration is a promising approach to limit habitat loss. More science is needed to increase the number of success stories 